\title{
Efficient Dynamic Computational Strategy for Heterogeneous Catalysis Based on Neural Network Potential Energy Surface: A Case Study of Temperature-Dependent Thermodynamics and Kinetics for the Chemisorbed on-surface $\mathrm{CO}$
}

\author{
Jun Chen, ${ }^{\dagger, \ddagger, \Phi, \perp}$ Tan Jin, ${ }^{\dagger, \ddagger}, \perp$ Tonghao Shen, ${ }^{,, \S}$ Mingjun Yang, ${ }^{*, \|}$ and Zhe-Ning \\ Chen ${ }^{*, \dagger, t, 9}$
}

$\dagger$ †ujian Science 83 Technology Innovation Laboratory for Optoelectronic Information of China, Fuzhou, Fujian 350108, P. R. China.

$\ddagger$ State Key Laboratory of Structural Chemistry, Fujian Institute of Research on the Structure of Matter, Chinese Academy of Sciences, Fuzhou 350002, P. R. China.

IState Key Laboratory of Physical Chemistry of Solid Surfaces, Fujian Provincial Key Laboratory of Theoretical and Computational Chemistry, College of Chemistry and Chemical Engineering, Xiamen University, Xiamen 361005, P. R. China.

$\S M O E$ Key Laboratory of Computational Physical Sciences, Department of Chemistry, Fudan University, Shanghai 200433, P. R. China.

\|Shenzhen Jingtai Technology Co., Ltd. (XtalPi), Floor 3, Sf Industrial Plant, No. 2 Hongliu Road, Fubao Community, Fubao Street, Futian District, Shenzhen 518045, P. R. China. $\perp$ These authors contributed equally to this work.

E-mail: shentonghao@fudan.edu.cn; mingjun.yang@xtalpi.com; znchen@fjirsm.ac.cn 


\begin{abstract}
As a favorable alternative and complement of experimental techniques, computational tools on top of ab initio calculations have played an indispensable role in revealing the molecular details, thermodynamics and kinetics in catalytic reactions. The static computational strategy, which recovers the reaction thermodynamics and kinetics based on the calculations of a few stationary geometries at zero temperature and some ideal statistic mechanics models, is the most popular approach in theoretical catalysis due to its simplicity. In comparison, the $a b$ initio molecular dynamics (AIMD) is a well-tested approach to provide more precise descriptions of catalytic processes, however, experiencing a significantly expensive computational cost in the direct $a b$ initio calculation of potential energy and gradients. Here we proposed a highly efficient dynamic computational strategy for the calculation of thermodynamic and kinetic properties in heterogeneous catalysis on the basis of neural network potential energy surface (NN PES) and MD simulations. Taking CO adsorbate on Ru(0001) surface as the illustrative model catalytic system, we demonstrated that our NN-PES-based MD simulations can efficiently generate the reliable smooth two-dimensional potentialof-mean-force (2-D PMF) surfaces in a wide range of temperatures (from 300 to 900 $\mathrm{K})$, and thus temperature-dependent thermodynamic properties can be obtained in a comprehensive investigation on the whole PMF surface rather than a rough estimation using ideal models based on a few optimized geometries. Moreover, MD simulations offer an effective way to describe the surface kinetics such as the CO adsorbate onsurface movement, which goes beyond the most popular static estimation based on calculated free energy barrier and transition state theory (TST). By comparing the results obtained in the dynamic and static approaches, we further revealed that the dynamic strategy significantly improves the predictions of both thermodynamic and kinetic properties as compared to the popular ideal statistic mechanics approaches such as harmonic analysis and TST. It is expected that this accurate yet efficient dynamic strategy can be a powerful tool in understanding reaction mechanisms and reactivity of a catalytic surface system, and further guides the rational design of heterogeneous
\end{abstract}


catalysts.

\section{Introduction}

The continuous demand for the sustainable development of our society requires the development of more active, more selective, and less expensive catalytic processes to eventually solve energy and environmental problems. Revealing the catalytic molecular mechanism and their corresponding statistical thermodynamic information is not only a central issue in catalytic science, but also provide a useful guide for the further rational design of the efficient catalytic processes. 1-4 Although an enormous amount of experimental effort has been conducted for molecular mechanism in heterogeneous catalysis, $5-8$ there is still experimental technical difficulties of obtaining molecular information directly. Theoretical and computational tools thus have become an indispensable approach for revealing the molecular details as well as the thermodynamics in catalysis.

Nowadays, theoretical calculations become really common in the field of catalysis. This, aligned with the rapid development in theoretical methods as well as the enormous growth in computational power. The static computational strategy is the most common approach

in theoretical catalysis, 2 in which only the limited stationary states at zero temperature are localized. The thermodynamics at specific conditions is computed by using the partition functions of ideal models. The ideal gas expressions are typically used for translational and rotational contributions of free molecules while the harmonic approximation is used for vibrational partition functions. This approach is computationally economical and thus has been widely used in theoretical catalysis. However, the reliability of this static approach depends on whether the realistic conditions significantly deviates from the ideal models. 2

The value of computational results for catalysis lies on whether the reliable computational thermodynamics at the specific condition could be obtained in theoretical approach. Notably, reaction temperature often plays a significant role in the activity as well as the 
selectivity for a catalytic reaction. $9-13$ Accurate calculation of the temperature-dependent thermodynamics in catalysis is thus critical for revealing the correct molecular mechanism and further guiding the rational design of more efficient catalytic processes. However, the degree of deviation between the realistic conditions and ideal models should closely relate to the temperature, which makes the reliability of temperature-dependent thermodynamics from the common static computational approach be doubted. Therefore, development an alternative computational strategy for theoretical catalysis that going beyond the common static computational strategy becomes necessary for calculating the reliable temperaturedependent thermodynamics.

Molecular dynamics (MD) is a well-tested approach to obtain the thermodynamics of the target system by going beyond static stationary states and sampling phase space more broadly.2,14,15 However, the computational cost for this dynamic computational strategy is significantly higher than the common static computational strategy. Catalytic processes generally involve breaking and formation of chemical bonds, clearly implies the necessity of employing ab initio electronic structure methods, instead of the more economical classical force fields, in the simulations, to provide a reasonably accurate description of the complicated electronic/chemical interactions. ab initio MD (AIMD) simulation is thus an appropriate choice for studies of catalysis, however, AIMD simulation achieves a reasonable length to provide meaningful thermodynamics is still challenging. Therefore, the most important aspect for using dynamic computational strategy in catalysis is to overcome the limitation of time scales in AIMD simulations. On one hand, proper enhanced sampling approaches could be introduced to allow an accelerated search in the phase space and thus fast thermodynamic properties evaluation. On the other hand, development of an efficient and accurate strategy to replace the direct ab initio calculations on system's energy and force is another approach to achieve a converged sampling more efficiently.

Various enhanced sampling strategies have been developed so far for accelerating phase space sampling, including but not limited to the approaches of umbrella sampling (US), 16 
replica exchange, 17,18 simulated tempering, 19,20 transition path sampling, 21 metadynamics (MTD),22,23 adaptive biasing force, ${ }^{24}$ temperature accelerated molecular dynamics (TAMD), $25-28$ and integrated tempering sampling (ITS),29,30 as well as some strategies of combining two enhanced sampling methods, such as ITS-MTD method developed by Gao et. al., 11 ITS-US and ITS-TAMD methods developed by us. 32,33 Recently, the enhanced sampling methods were combined with AIMD simulations to solve some issues in heterogeneous catalysis. 2,34,35 It's worth mentioning that we have provided a dynamic computational strategy by combining ITS method and AIMD simulation for the temperature-dependent thermodynamics of $\mathrm{CO}$ diffusion on $\mathrm{Ru}(0001)$ surface. 35 Although the enhanced sampling method indeed significantly improves the sampling efficiency, the computational cost is still awfully expensive for achieving a converged sampling. Limited thermodynamic information only at three temperature was gave in our previous ITS enhanced AIMD simulations. Therefore, we have been aware of that such enhanced AIMD based dynamic computational strategy is still too expensive to face the realistic issues in catalysis. Further reducing the cost in calculation of system's energy and force is necessary.

Construction of the accurate global potential energy surface (PES) could avoid the expensive direct $a b$ initio calculations. Actually, an accurate PES is essential for the theoretical research in chemical reaction dynamics. In the past few decades, development of fitting techniques of $a b$ initio electronic structure data has made the construction of accurate global PESs possible for multidimensional molecular systems. $\frac{36}{}$ More recently, the artificial neural network (NN) methods have displayed powerful in construction of the complex PES, which promotes the wide utilization of NN PES on the chemical reaction dynamics for multidimensional molecular systems. $37-40$ The NN PES also gives hope for dealing with the more realistic chemical issues. Actually, some limited NN PES MD simulations have appeared in the material and catalysis science. 41

The adsorption and activation of CO molecule on a transition metal surface is often a critical elementary step for the catalytic conversion of CO into the high added-value chemicals, 
such as Fischer-Tropsch reaction, $42,44 \mathrm{CO}_{2}$ reduction, 45,47 and coal to ethylene glycol, 18,50 etc. Given the fact that there exist many active sites on the surface, presumably with different binding affinity to the substrates, intermediates, as well as transition states, a proper description of the adsorbate adsorption and movement between different sites on surface is thus clearly important for a correct understanding of the catalytic process. It is worth noting that $\mathrm{CO}$ molecule adsorbed on a transition metal surface is a prototype system for molecular chemisorption, 51 and the diffusion of $\mathrm{CO}$ molecule on metal surfaces in fact involves breaking and formation of metal-carbon bonds, very much like a typical chemical reaction process. This fact clearly implies the economical classical force fields cannot make a reliable description for the adsorption and movement for $\mathrm{CO}$ on surface.

In this contribution, we proposed an accurate and efficient dynamic strategy for the temperature-dependent thermodynamics of heterogeneous catalysis, in which the NN PES was constructed beforehand instead of the direct ab initio calculations. Using CO adsorbate on $\mathrm{Ru}(0001)$ surface as the illustrative model catalytic system, our NN PES MD simulations obtained the accurate temperature-dependent thermodynamics efficiently and generated the reliable smooth two-dimensional potential-of-mean-force (2-D PMF) surfaces in a wide range of temperatures that from 300 to $900 \mathrm{~K}$. By proper considering the thermodynamic contribution from the adsorbate in-plane motions that was missed when generating the 2-D PMF surfaces, the free energy difference as well as the diffusion barrier between the two stable adsorption sites (top and $h c p$ ) were unexpected found to increase rather than reduce with temperature raising. Benefit from the high efficiency for our proposed dynamic strategy, the temperature-dependent kinetics for the adsorbate on-surface movement can be calculated directly from the converged sampling trajectories, which provides a chance to evaluate the validity of transition state theory (TST) that is the most common approach for kinetics calculation in theoretical catalysis. Our calculations indicated there is significant quantitatively deviation for TST in the adsorbate on-surface kinetics. Finally, we made a careful comparison between the proposed dynamic computational strategy and the common static 
computational strategy, and further revealed the limitation of static computational strategy in temperature-dependent thermodynamics. Our study clearly demonstrates the efficiency and reliability of the dynamic computational strategy based on NN PES and MD simulations, which is expected as a powerful tool for the statistical thermodynamics and kinetics calculation in catalysis, in conjunction with the proper enhanced sampling approaches when necessary.

\section{Computational Methods}

\subsection{Density Functional Theory}

All the potential energies were calculated using Perdew-Burke-Ernzerhof (PBE) functional 52 with the VASP (Vienna ab initio Simulation Package) code, $\underline{53,54}$ using the projector-augmentedwave $(\mathrm{PAW})^{55}$ method together with plane-wave basis sets to describe the electron-ion interactions. The kinetic energy cutoff was set to $400 \mathrm{eV}$. The convergence criteria for the energy calculations in self-consistent field was set to $1.0 \times 10^{-5} \mathrm{eV}$. Integration over the Brillouin zone was performed by using the Monkhorst-Pack scheme 56 with $3 \times 3 \times 1 k$-points, together with a Gaussian smearing broadening of $0.1 \mathrm{eV}$.

\subsection{Data Sampling and Potential Energy Surface}

The PES was constructed with NN fitting to a data set sampled in the six dimensional space, which has the following functional form

$$
y=b^{3}+\sum_{k=1}^{K}\left(w_{k}^{3} \cdot f^{2}\left(b_{k}^{2}+\sum_{j=1}^{J}\left(w_{j, k}^{2} \cdot f^{1}\left(b_{j}^{1}+\sum_{i=1}^{I}\left(w_{i, j}^{1} \cdot x_{i}\right)\right)\right)\right)\right) .
$$

in which $x_{i}$ is the coordinate vector of a configuration, and $y$ is the corresponding potential energy. $I$ corresponds to the dimension of the PES, while $J$ and $K$ determine the structure of a NN function. $w_{i, j}^{1}, w_{j, k}^{2}, w_{k}^{3}, b_{j}^{1}, b_{k}^{2}$, and $b^{3}$ are variable parameters of a $\mathrm{NN}$ function, 
which can be determined by training algorithms. With the activation function of two hidden layers $f^{1}$ and $f^{2}$ chosen to be tanh, and noting the output of each neuron in hidden layers as $y_{j}^{1}$ and $y_{k}^{2}$, the gradient of potential energy, which is necessary for MD silulation, can be obtained analytically by

$$
\frac{d y}{d x_{i}}=\sum_{k=1}^{K}\left(w_{k}^{3} \cdot\left(1-\left(y_{k}^{2}\right)^{2}\right) \cdot \sum_{j=1}^{J}\left(w_{j, k}^{2} \cdot\left(1-\left(y_{j}^{1}\right)^{2}\right) \cdot w_{i, j}^{1}\right)\right)
$$

The lattice of $\mathrm{Ru}(0001)$ was simulated with 5 atom layers and $2 \times 2$ supercell, results in 20 $\mathrm{Ru}$ atoms in a cell. The optimized lattice constant of $2.740 \AA$ was used. The CO molecule was scattered on the rigid $\mathrm{Ru}(0001)$ surface, with the height of geometry center of $\mathrm{CO}$ molecule to the surface ranging from 1.4 to $7.5 \AA$. All the configurations were mapped to the symmetrically unique intervals, as illustrated by the orange triangle in Figure S1. Bond length of CO molecule ranges from 0.95 to $1.45 \AA$, which limits the potential energy of free CO molecules to less than $3.5 \mathrm{eV}$.

The data set was firstly constructed with about 10000 configurations obtained from the ab initio dynamics reported in 2015. 35 A primitive set of NN PESs can be obtained with NN fittings to the initial data set. After that a large number of quasi-classical dynamics trajectories have been calculated on the PES. From these trajectories, those configurations which show large deviation in potential energies obtained from different primitive NN PESs are further calculated with DFT and added to the data set. The NN fittings, quasi-classical dynamics, as well as DFT calculations were repeated iteratively. This configuration selection scheme has been proved to be efficient in PES construction for both gas phase reactions and gas-surface interaction systems. 40,57 In total 56007 configurations have been selected finally for PES construction. (see Figure S2 for the potential energy distribution of all the configurations). With a NN function of 6-40-40-1, the global PES has a total fitting error of $3.82 \mathrm{meV}$. 


\subsection{Molecular Dynamics Simulation and Thermodynamics Calcu- lation}

The Molecular dynamics (MD) simulations were carried out in the canonical ensemble

from 300 to $900 \mathrm{~K}$, with the Nosé-Hoover thermostat 58,59 for temperature controlling. The velocity-verlet algorithm 60,61 was employed to update the velocities and coordinates with a time step of $0.5 \mathrm{fs}$. The energy and force were obtained directly from the NN PES. The 2-D PMF surfaces were generated from the MD trajectories using the two in-plane coordinates of the carbon atom as the reaction coordinates. It is worth noting that the thermodynamic contribution of the two in-plane modes of adsorbate is missed in a 2-D PMF surface scheme. Therefore, relative free energy for the adsorbate on a specified on-surface site was evaluated by considering the occupation probability of adsorbate not only on a local minimum point but also within a region near degeneracy in free energy (setting as $1 \mathrm{kcal} / \mathrm{mol}$ in this work) around the local minimum point. Similarly, the free energy barrier for adsorbate diffusion was evaluated by considering the occupation probability of adsorbate on a 2-D area of the PMF with the close free energy (within $1 \mathrm{kcal} / \mathrm{mol}$ above the transition state, illustrated as Figure S3 in the supporting material). The rate constant of barrier corssing $k$ is evaluated by TST as

$$
k=\frac{k_{\mathrm{B}} T}{h} \exp \left[-\frac{\Delta_{r}^{\neq} G_{m}^{\varnothing}}{R T}\right]
$$

where $\Delta_{r}^{\neq} G_{m}^{\varnothing}$ is the free energy barrier.

In addition, the rate constants have also been directly calculated from the diffusion time of MD trajectories. The diffusion time of a diffusion process was recorded from the trajectory's first entering of the top region to it's first entering of the hcp region. A typical diffusion process was shown in Figure S4. The diffusion rates were directly calculated from the average diffusion time by

$$
k=\frac{1}{t_{\text {average }}} .
$$

As a comparison, the common static computational strategy was also conducted for 
thermodynamic properties evaluation. The static structure calculations were carried out with a maximum Hellmann-Feynman force tolerance of $0.01 \mathrm{eV} / \AA$. The climbing image nudged-elastic band (CI-NEB) method was utilized to locate the transition state structure. Numerical calculation of the second derivatives of the transisiton state provided the harmonic vibrational frequencies and corresponding normal modes. A geometrical displacement of 0.01 $\AA$ was used for all vibrational calculations.

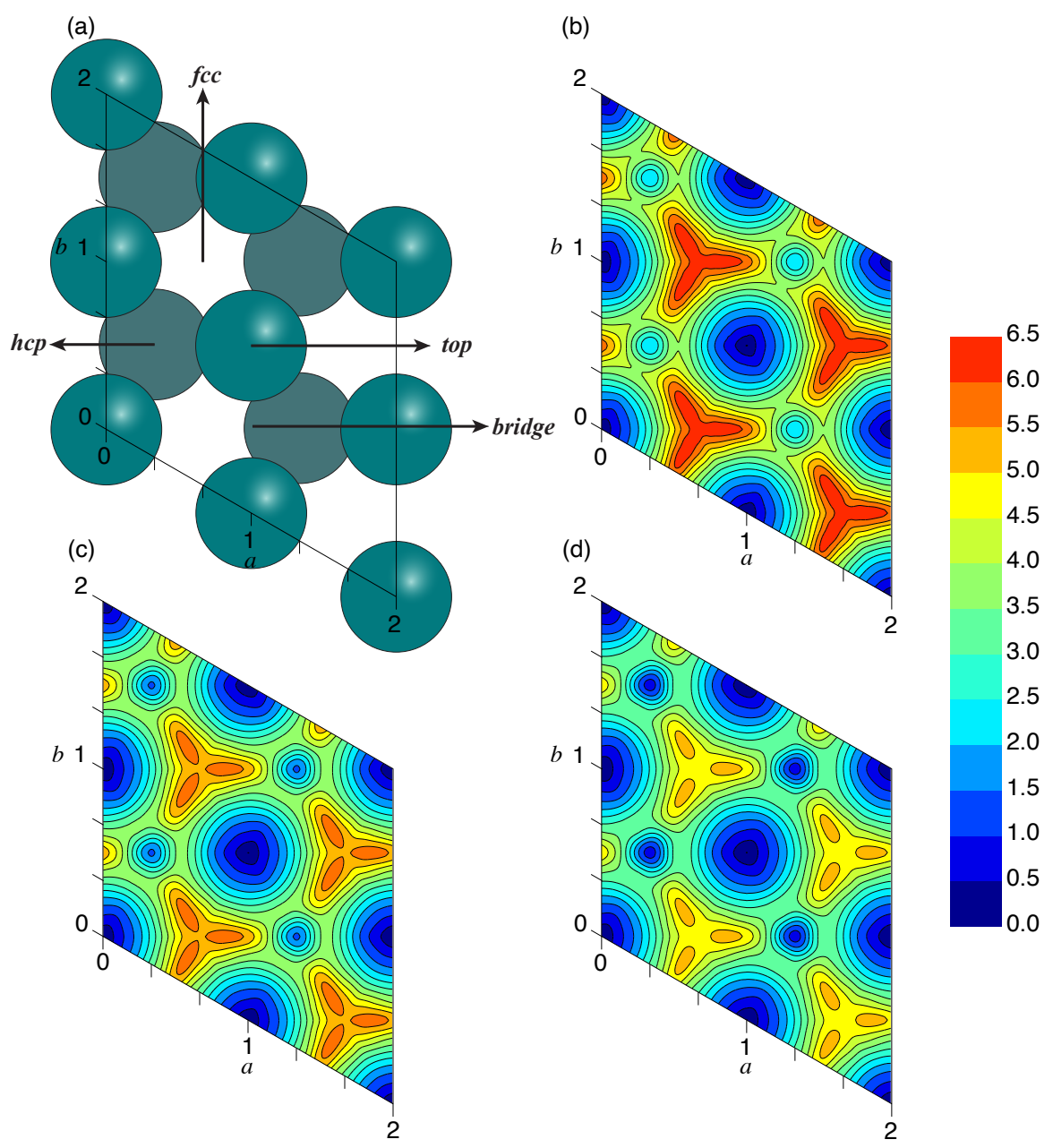

Figure 1: Model of $\mathrm{Ru}(0001)$ surface and 2-D PMF surfaces of $\mathrm{CO}$ adsorbate on the $\mathrm{Ru}(0001)$ surface along the fractional coordinates $a$ and $b$ of carbon atom on the surface plane. (a) $\mathrm{Ru}(0001)$ surface with a $2 \times 2$ supercell in the lateral directions; (b) PMF at $300 \mathrm{~K}$; (c) PMF at $600 \mathrm{~K} ;(\mathrm{d}) \mathrm{PMF}$ at $900 \mathrm{~K}$. 


\section{Results}

\subsection{2-D PMF surfaces at different temperatures.}

The highly efficient in the current NN PES for calculating the interaction between the adsorbate and surface makes it possible to achieve the converged sampling by a long length simulation. The temperature-dependent thermodynamics were obtained from MD simulations for a total time of $3.5 \mu$ s at each of seven different temperatures, ranging from 300 to $900 \mathrm{~K}$. The obtained 2-D PMF surfaces for CO adsorbate on $\mathrm{Ru}(0001)$ surface are illustrated in Figure 1 and Figure S5 with respect to the fractional coordinates $a$ and $b$ of the carbon atom of $\mathrm{CO}$ adsorbate on the surface plane.

The PMF surfaces clearly show the existence of two distinct thermodynamically stable CO binding sites, top and hcp. The top site is more favored than $h c p$ site at all the simulated

temperatures. This agrees with the previous experimental and theoretical results, 62,63 and confirms the reliability of this newly reported PES. As shown in Figure 1 and Figure S5, with increasing temperature, the 2-D PMF surfaces grow flatter and flatter, which means the distribution of $\mathrm{CO}$ adsorbate at different surface sites becomes more and more uniform, agreeing with the observed increased presence of $\mathrm{CO}$ at higher coordination sites with temperature raising in the previous spectroscopic experiments. 64 66

\subsection{Temperature-dependent thermodynamics for CO adsorbate on the stable binding sites of surface.}

The PMF surfaces show that there are two stable CO binding sites, top and hcp, on the $\mathrm{Ru}(0001)$ surface. First, we quantitatively describe the binding free energy difference between the two sites by only considering the local minimum point on top and $h c p$ sites, respectively. As shown in the blue line of Figure 2a (noted as the "PMF point" approach), the calculated binding free energy difference between top and $h c p$ sites becomes smaller as temperature increases, in line with the observed PMF surfaces. However, the thermodynamic contribution 


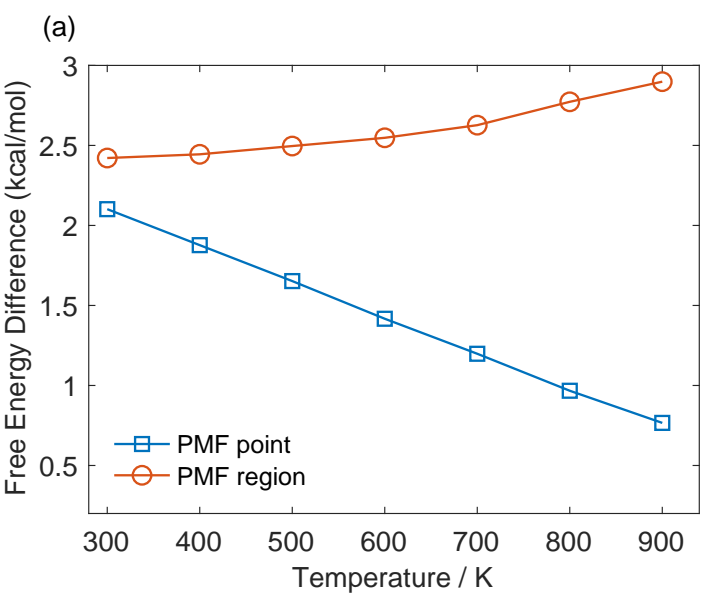

(b)

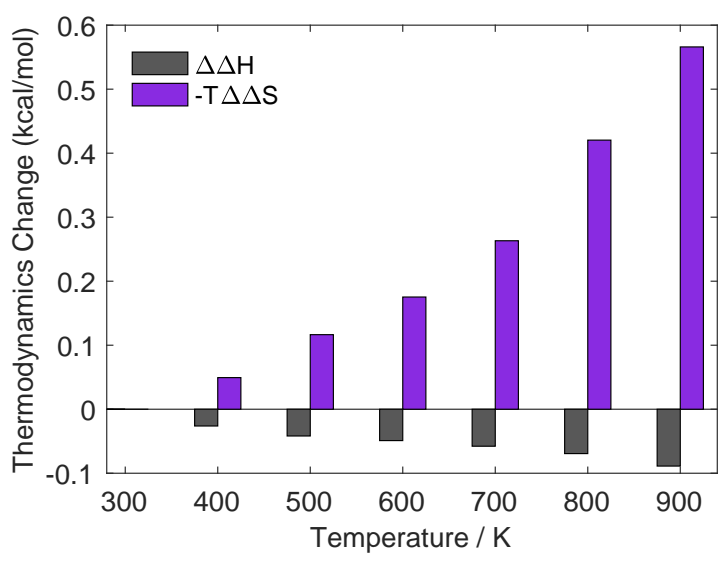

Figure 2: Temperature-dependent (a) free energy difference and (b) corresponding contribution from enthalpy and entropy between top and $h c p$ sites calculated based on PMF surfaces.

of in-plane modes of adsorbate has been missed if only the local minimum point in 2-D PMF surface was considered. Instead, the missed contribution from the in-plane modes could be largely included when the occupation probability was calculated by considering $\mathrm{CO}$ adsorbate in a region other than a single local minimum point. Therefore, the relative adsorption free energy on a stable binding site was evaluated based on the local minimum point with additional near degeneracy region (within $1 \mathrm{kcal} / \mathrm{mol}$ ) around the point. As shown in Figure 1, the near degeneracy region in top site is obviously larger than that in $h c p$ site, suggesting the more freeness for $\mathrm{CO}$ adsorbate movement on the top site.

The distribution of $\mathrm{CO}$ adsorbate at top and $h c p$ regions still becomes more and more 
uniform with the rising of temperature (see Table S1). However, it is unexpected to find that their variation of relative free energy exhibits an opposite trend. As shown in the red

line of Figure 2a (noted as the "PMF region" approach), the free energy difference between top and $h c p$ regions increases as temperature raising, in contrary to the intuition. Further analysis of the contribution from enthalpy and entropy revealed that, as shown in Figure $2 \mathrm{~b}$, the entropic effect plays an important role in the temperature-dependent free energy change. As the more freeness for adsorbate movement on the top site, adsorption of $\mathrm{CO}$ molecule on top site should be facilitated by entropic effect, resulting in the observed unexpected temperature-dependent thermodynamic properties.

\subsection{Diffusion kinetics of CO adsorbate between the two stable binding sites on surface.}

Diffusion of adsorbates is the primary means for accomplishing two crucial events required for catalysis: the encounter of different reaction partners to form the reactant complex and the arrival at an active site which provides strong affinity for the active intermediate of the reaction. The PMF surfaces clearly show significant barriers separating different binding sites. The diffusion of $\mathrm{CO}$ adsorbate should quite likely follow a typical jumping-amongminima behavior, making an elementary process in surface catalysis. The diffusion kinetics could be obtained based on the calculated free energy barrier in conjunction with TST, likely the most common approach in theoretical catalysis. Moreover, we would like to mention another approach to calculate the diffusion kinetics based on the dynamic protocol proposed here, that is, calculating the diffusion rate constant $(k)$ directly from the average diffusion time of MD trajectories jumping from one adsorption site to another. Based on adequate sampling, such approach undoubtedly possesses a more accuracy since the intrinsic error of TST can be properly avoided.

As mentioned above, definition of a proper region around the local minimum point is necessary for evaluating the thermodynamics for a specified binding site. Similarly, defi- 

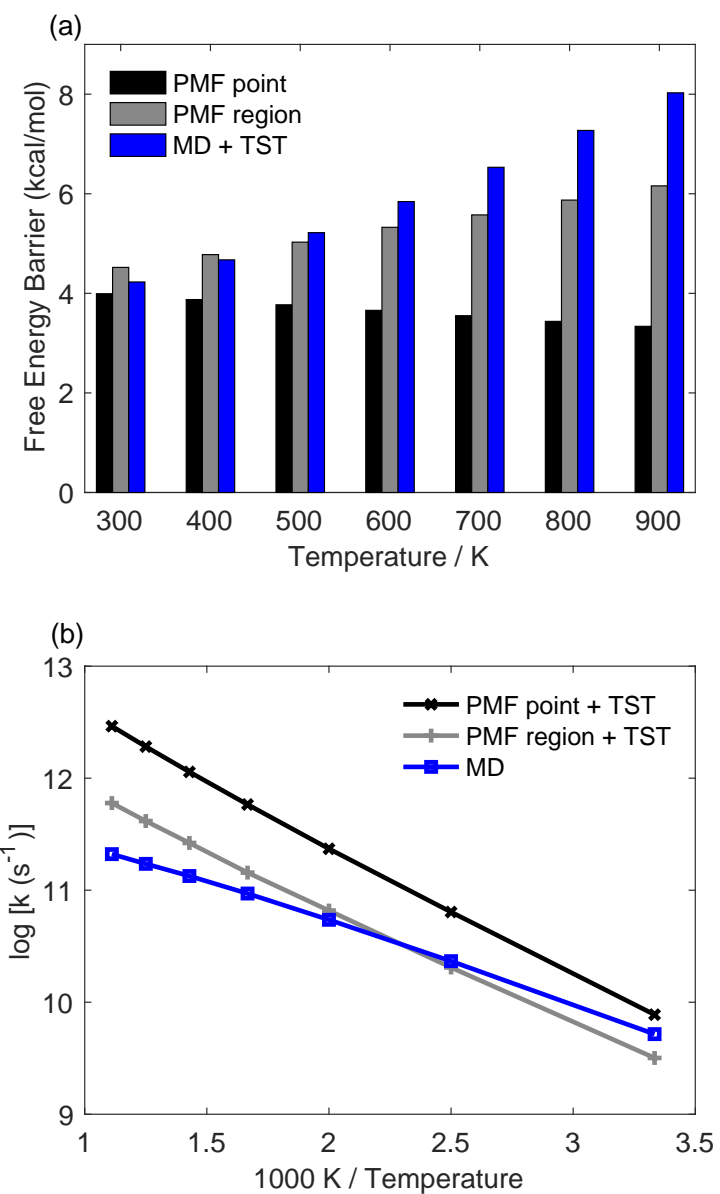

Figure 3: Temperature-dependent (a) free energy barriers and (b) rate constants for CO adsorbate diffusion from top to hcp region calculated based on PMF surfaces and diffusion time of MD trajectories, respectively.

nition of a proper region around the saddle point is necessary for calculating the kinetics. Accordingly, the diffusion barrier can be calculated based on the occupation probability difference of $\mathrm{CO}$ adsorbate between the defined TS region and the corresponding local minimum region. The calculated diffusion barriers based on PMF surfaces for CO adsorbate moving from top to $h c p$ region were illustrated as gray bars in Figure 3 a. The corresponding diffusion rate constants $(k)$ can be further evaluated based on TST and were illustrated as a gray line in Figure 3b, noted as the "PMF region + TST" approach. As a comparison, the free energy differences between TS and top sites displayed on 2-D PMFs were shown as 
black bars in Figure $3 a$. On the other hand, the diffusion rate constants were calculated directly from the average diffusion time of MD trajectories, and illustrated as a blue line in Figure 3b, noted as the "MD" approach. The corresponding diffusion free energy barriers can be backstepped based on TST and illustrated as blue bars in Figure 3a, noted as the "MD + TST" approach. Our results showed that the diffusion barriers calculated by the "PMF region" and "MD + TST" approaches both increase with the rise of temperature, in consistent with the variation of relative free energy between top and $h c p$ regions with temperature change shown in Figure 2a. However, there is significant difference in high temperature limit for the calculated kinetics by these two approaches, which can be mostly attributed to the limitation of TST. As shown in Figure $3 a$ and Table S2, we took the absolute percentage difference (APD) to evaluate the deviation in the backstepped free energy barrier from TST based on the calculated $k$ by the "MD" approach. As shown, the APD is as high to $30.4 \%$ at $\mathrm{T}=900 \mathrm{~K}$, and is showed to reduce along with the decreasing of temperature. When temperature drops below $500 \mathrm{~K}$, the APD is reduced to within $10 \%$. Meanwhile, As shown in Figure $3 \mathrm{~b}$ and Table S2, the deviation of $k$ based on the free energy barrier and TST was also evaluated. As shown, the TST underestimated the $k$ by $38.8 \%$ at $300 \mathrm{~K}$, and increased dramatically to $184.4 \%$ when temperature is raised to $900 \mathrm{~K}$. In contrast, the free energy differences (noted as "PMF point" approach in Figure 2a) show an opposite trend with the change of temperature, mainly due to the lack of two in-plane modes considered in free energy calculations. It is also found in Figure $3 \mathrm{~b}$ that, different to TST rates based on PMF barriers, the directly calculated rates from diffusion time of MD trajectories show an obvious non-Arrhenius property, which should be close to the practical motif of an elementary process in heterogeneous catalysis. $\underline{67,68}$

\subsection{Comparison of the dynamic and static strategies.}

The static computational strategy, which is based on the relative potential energy and harmonic vibrational frequencies of an optimized transition state, is the most common strategy 

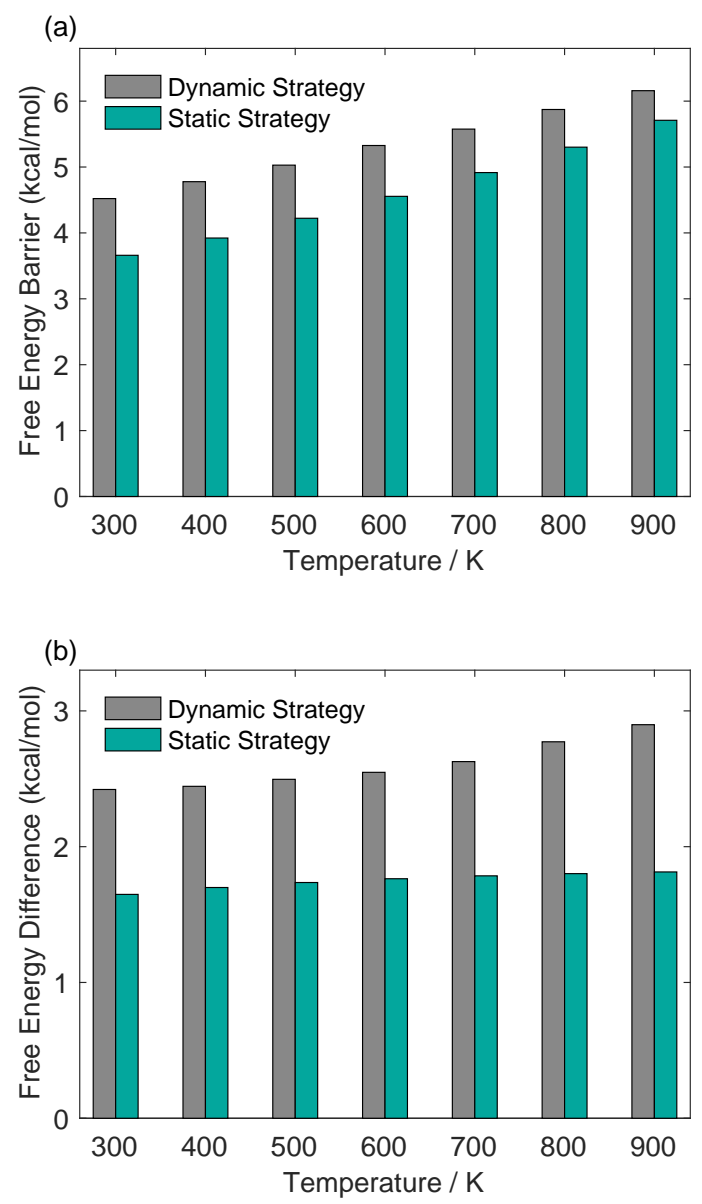

Figure 4: Temperature-dependent (a) free energy barrier and (b) free energy difference for $\mathrm{CO}$ adsorbate diffusion from top to $h c p$ region calculated based on dynamic strategy (the "PMF region" approach) and static strategy, respectively.

in theoretical catalysis. However, its reliability depends on the degree of the realistic conditions deviation from the ideal models. Here the benchmark results from dynamic computational strategy provide us a chance to go beyond the ideal model so that reach a higher accuracy compared to statistic thermodynamic properties. As shown in Figure 4 and Table S3, although the variation tendency for the temperature-dependent thermodynamics between static and dynamic computational strategies is consistent, the static strategy significantly underestimates both the relative free energy and diffusion barrier between top and hcp regions. Our calculations indicated the APD for the calculated relative free energy from static 
strategy against that from dynamic strategy are in the range of $30 \%$ to $37 \%$. The difference of calculated free energy barriers shown in Figure ta will introduce APD of $159.4 \%$ to 265.6\% in the diffusion rate, as listed in Table S3. Moreover, the deviation for static strategy are shown to increase along with the raising of temperature. At the high temperature limit of 900 K, result from static strategy cannot even reach chemical accuracy. These findings suggest that, there is indeed significant deviation for description of the adsorption and movement for adsorbate on surface by using the common static strategy, especially in a high temperature.

\section{Discussion}

In the computational investigation on catalysis, the static computational strategy is the most common approach, in which the limited stationary states at zero temperature are localized and subsequently based on the partition functions of ideal models to obtain the thermodynamics at the realistic experimental conditions. Therefore, the reliability of static computational strategy depends on whether the ideal models are deviated from the realistic conditions. In comparison, MD simulations is a well-tested approach to obtain the thermodynamics as well as the kinetics of the target system by going beyond ideal models. The reliability of the dynamic computational strategy in statistical thermodynamics depends on whether the converged sampling can be achieved.

The dynamic computational strategy based on AIMD simulations has been used to face

some issues in heterogeneous catalysis. 2 However, the expensive computational cost in AIMD simulations greatly limited its wide applications. The NN PES provides an accurate and efficient computational approach by avoiding most expensive $a b$ initio calculations. Therefore, a dynamic computational strategy by combination of NN PES and MD simulations has been proposed here for efficient evaluation of temperature-dependent thermodynamics for heterogeneous catalysis. We have constructed a 6-D NN PES for CO adsorbate on Ru(0001) surface, which can fully reproduce the potential energy and gradients at $a b$ initio level. 
Base on it, converged sampling by MD simulation has been carried out for the temperaturedependent thermodynamics of $\mathrm{CO}$ adsorption and diffusion on the surface. Benefit from our highly efficient dynamic computational strategy, the temperature-dependent thermodynamics have been obtained from a total of $3.5 \times 10^{3}$ ns long MD simulations at each of seven temperatures, that is $300 \sim 900 \mathrm{~K}$.

We obtained the smooth 2-D PMF surfaces for $\mathrm{CO}$ adsorbate on $\mathrm{Ru}(0001)$ surface at 300 $\sim 900 \mathrm{~K}$ (see Figure 1 and S5), displaying the intuitive pictures of temperature-dependent thermodynamics. However, the thermodynamic contribution of adsorbate in-plane motion is missed when the phase space is projected to a 2-D PMF surface. We thus proposed here using a properly defined region other than a single local minimum (or saddle) point to include the contribution of adsorbate in-plane motion. Our results unexpectedly showed that the relative free energy between top and $h c p$ sites increases rather than reduces as the temperature raising, being contrary to the intuition (see Figure 2). The PMF surfaces in Figure 1 and S5 obviously suggest that the free energy curve in top site is flatter than that in $h c p$ site, in agreement with our previous results that the frequency of the two in-plane modes is lower in the top site,, 35 and further suggests the top site has less steric interactions. Therefore, the in-plane motion is easier in the top site, which makes the adsorption and movement of $\mathrm{CO}$ molecule on top site is more facilitated by entropic effect, resulting in the observed counterintuitive temperature-dependent thermodynamic properties.

In the common computational treatment in theoretical catalysis, the reaction kinetics are mostly obtained from the combination of calculated free energy barrier and TST. Our proposed efficient dynamic computational strategy can not only provide the free energy barrier by a proper statistical protocol, but also calculate the diffusion rate constant $(k)$ directly from the average diffusion time of MD trajectories. It means the evaluation of kinetics is not necessary to rely on the TST in our newly provided dynamic computational strategy, which provides a benchmark to evaluate the validity of TST. As shown in Figure 3 and Table S2, there is significant deviation for the calculated kinetics in TST, indicating the limitation of 
TST for reaction kinetics, or at least for the on-surface diffusion processes. Our calculations showed the deviation for TST is particularly significant at high temperature. Such as, the APD for diffusion barrier is as high to $30.4 \%$, and the rate constant $k$ is even overestimated by an APD value of $184 \%$ at $900 \mathrm{~K}$. The deviation of TST is shown to reduce along with the drop of temperature. It has been proved again that TST is improper for the kinetics of low barrier process, further revealing the necessity for using dynamic computational strategy in direct evaluation of the more accurate catalytic kinetics in quantitative. It is also suggested that the non-Arrhenius behavior of an elementary process, which should be universal in dynamics, can only be accessed by the direct computation of $k$ from the average diffusion time of MD trajectories.

Our proposed dynamic computational strategy also provides a chance to evaluate the reliability of the common static computational strategy. As shown in Figure 4 and Table S3, there is significant deviation for static computational strategy in quantitative, in which the APD for the calculated thermodynamics and kinetics are within the range of $30 \%$ to $37 \%$ and $157 \%$ to $266 \%$, respectively, revealing the limitation of ideal statistical models. We are pleased to find that there is consistent variation tendency for the temperature-dependent thermodynamics between static and dynamic computational strategies, indicating the applicability of static computational strategy in qualitative for such simple case. However, the deviation of static computational strategy is expected to be further increased when faced with the more complex realistic catalytic systems. The dynamic computational strategy thus become necessary if the quantitatively accurate description for catalytic processes and the further catalyst rational design are required.

\section{Conclusion}

In summary, we have proposed an efficient dynamic computational strategy for catalysis based on NN PES MD simulations. The 6-D NN PES for a model system of heterogeneous 
catalysis, that is $\mathrm{CO}$ adsorbate on $\mathrm{Ru}(0001)$ surface, has been constructed. Based on this, longtime MD simulations have been carried out for the temperature-dependent thermodynamics of adsorbate adsorption and movement on surface. The smooth and reliable 2-D PMF surfaces at seven different temperatures (from 300 to $900 \mathrm{~K}$ ) have been obtained, and abundant temperature-dependent thermodynamic properties have archived. To the best of our knowledge, this is the first report of NN PES MD simulations on temperature-dependent thermodynamics for a chemisorbed molecule on transition metal surface.

The temperature-dependent thermodynamics as well as the diffusion kinetics for adsorbate on-surface adsorption and movement have been explored by this dynamic computational strategy. We are unexpected to find that the calculated free energy difference and diffusion barrier between the two stable binding sites (top and $h c p$ ) increase other than reduce along with the temperature raising, contrary to the common intuition. In addition, our dynamic computational strategy provides an efficient approach to evaluate the reaction kinetics directly, and also provides a benchmark to evaluate the applicable of TST that is the most common computational tool for obtaining reaction kinetics in theoretical catalysis. Our results also showed that there is significant deviation for TST in on-surface diffusion kinetics.

Our dynamic computational strategy also provides a chance to evaluate the reliability of common static computational strategy. Our results indicated there is significant deviation of static computational strategy, although the static computational strategy performs a right variation tendency with temperature change in qualitative for this selected simple case. It can be expected that the deviation of static computational strategy would be further increased when faced with the more complex realistic catalytic systems. These findings demonstrated the necessity for usage of dynamic computational strategy to obtain the quantitatively accurate information in catalysis. Our study clearly indicated that the dynamic computational strategy based on NN PES MD simulations is readily available as an efficient and reliable approach to study the thermodynamics and kinetics of catalysis. By further combination of the advanced density functional approximations 69 as well as the proper enhanced sampling 
protocols, $31-33$ the proposed dynamic computational strategy is expected to open an avenue for accurate and efficient operando computational modeling for heterogeneous catalysis.

\section{Acknowledgments}

This work is financially supported by Fujian Science \& Technology Innovation Laboratory for Optoelectronic Information of China (2021ZR109), the Natural Science Foundation of Fujian Province (2019J01131), the National Natural Science Foundation of China (21973094, 22173104, 22173105), and the Opening Project of PCOSS of Xiamen University (201908).

\section{References}

(1) Norskov, J. K.; Bligaard, T.; Rossmeisl, J.; Christensen, C. H. Towards the computational design of solid catalysts. Nat. Chem. 2009, 1, 37-46.

(2) Grajciar, L.; Heard, C. J.; Bondarenko, A. A.; Polynski, M. V.; Meeprasert, J.; Pidko, E. A.; Nachtigall, P. Towards operando computational modeling in heterogeneous catalysis. Chem. Soc. Rev. 2018, 47, 8307-8348.

(3) Li, L. L.; Chang, X.; Lin, X. Y.; Zhao, Z. J.; Gong, J. L. Theoretical insights into single-atom catalysts. Chem. Soc. Rev. 2020, 49, 8156-8178.

(4) Jimenez-Izal, E.; Alexandrova, A. N. Computational Design of Clusters for Catalysis. Annu. Rev. Phys. Chem. 2018, 69, 377-400.

(5) Wang, X.; Huang, S. C.; Huang, T. X.; Su, H. S.; Zhong, J. H.; Zeng, Z. C.; Li, M. H.; Ren, B. Tip-enhanced Raman spectroscopy for surfaces and interfaces. Chem. Soc. Rev. 2017, 46, 4020-4041.

(6) Guo, Q.; Zhou, C. Y.; Ma, Z. B.; Ren, Z. F.; Fan, H. J.; Yang, X. M. Elementary 
Chemical Reactions in Surface Photocatalysis. Annu. Rev. Phys. Chem. 2018, 69, $451-472$.

(7) Magnussen, O. M.; Gross, A. Toward an Atomic-Scale Understanding of Electrochemical Interface Structure and Dynamics. J. Am. Chem. Soc. 2019, 141, 4777-4790.

(8) Bergmann, U.; Kern, J.; Schoenlein, R. W.; Wernet, P.; Yachandra, V. K.; Yano, J. Using X-ray free-electron lasers for spectroscopy of molecular catalysts and metalloenzymes. Nat. Rev. Phys. 2021, 3, 264-282.

(9) Bond, G. C.; Keane, M. A.; Kral, H.; Lercher, J. A. Compensation phenomena in heterogeneous catalysis: General principles and a possible explanation. Catal. Rev. 2000, 42, 323-383.

(10) Hagen, C. M.; Widegren, J. A.; Maitlis, P. M.; Finke, R. G. Is it homogeneous or heterogeneous catalysis? Compelling evidence for both types of catalysts derived from $\left[\mathrm{Rh}\left(\eta^{5}-\mathrm{C}_{5} \mathrm{Me}_{5}\right) \mathrm{Cl}_{2}\right]_{2}$ as a function of temperature and hydrogen pressure. J. Am. Chem. Soc. 2005, 127, 4423-4432.

(11) Li, W.; Yang, H.; Chen, S.; Chen, Q.; Luo, L.; Li, J.; Chen, Y.; Li, C. TemperatureDependent Morphology Evolution of Boron Nitride and Boron Carbonitride Nanostructures. J. Nanomater. 2019, 2019.

(12) Hou, J.; Yang, M.; Zhang, J. Recent advances in catalysts, electrolytes and electrode engineering for the nitrogen reduction reaction under ambient conditions. Nanoscale 2020, 12, 6900-6920.

(13) Li, Z.; Ji, S.; Liu, Y.; Cao, X.; Tian, S.; Chen, Y.; Niu, Z.; Li, Y. Well-Defined Materials for Heterogeneous Catalysis: From Nanoparticles to Isolated Single-Atom Sites. Chem. Rev. 2020, 120, 623-682. 
(14) Chipot, C. Frontiers in free-energy calculations of biological systems. WIREs Comput. Mol. Sci. 2014, 4, 71-89.

(15) Bruix, A.; Margraf, J. T.; Andersen, M.; Reuter, K. First-principles-based multiscale modelling of heterogeneous catalysis. Nat. Catal. 2019, 2, 659-670.

(16) Kastner, J. Umbrella sampling. WIREs Comput. Mol. Sci. 2011, 1, 932-942.

(17) Swendsen, R. H.; Wang, J. S. Replica Monte Carlo Simulation of Spin-Glasses. Phys. Rev. Lett. 1986, 57, 2607-2609.

(18) Sugita, Y.; Okamoto, Y. Replica-exchange molecular dynamics method for protein folding. Chem. Phys. Lett. 1999, 314, 141-151.

(19) Lyubartsev, A. P.; Martsinovski, A. A.; Shevkunov, S. V.; Vorontsovvelyaminov, P. N. New approach to Monte Carlo calculation of the free energy: Method of expanded ensembles. J. Chem. Phys. 1992, 96, 1776-1783.

(20) Marinari, E.; Parisi, G. Simulated Tempering: A New Monte Carlo Scheme. Europhys. Lett. 1992, 19, 451-458.

(21) Bolhuis, P. G.; Chandler, D.; Dellago, C.; Geissler, P. L. Transition path sampling: Throwing ropes over rough mountain passes, in the dark. Annu. Rev. Phys. Chem. 2002, 53, 291-318.

(22) Laio, A.; Parrinello, M. Escaping free-energy minima. Proc. Nat. Acad. Sci. USA 2002, 99, 12562-12566.

(23) Li, H.; Min, D.; Liu, Y.; Yang, W. Essential energy space random walk via energy space metadynamics method to accelerate molecular dynamics simulations. J. Chem. Phys. 2007, 127, 094101.

(24) Darve, E.; Rodriguez-Gomez, D.; Pohorille, A. Adaptive biasing force method for scalar and vector free energy calculations. J. Chem. Phys. 2008, 128, 144120. 
(25) Gu, W.; Wang, T. T.; Zhu, J.; Shi, Y. Y.; Liu, H. Y. Molecular dynamics simulation of the unfolding of the human prion protein domain under low $\mathrm{pH}$ and high temperature conditions. Biophys. Chem. 2003, 104, 79-94.

(26) Maragliano, L.; Vanden-Eijnden, E. A temperature accelerated method for sampling free energy and determining reaction pathways in rare events simulations. Chem. Phys. Lett. 2006, 426, 168-175.

(27) Maragliano, L.; Vanden-Eijnden, E. Single-sweep methods for free energy calculations. J. Chem. Phys. 2008, 128, 184110.

(28) Hu, Y.; Hong, W.; Shi, Y.; Liu, H. Temperature-Accelerated Sampling and Amplified Collective Motion with Adiabatic Reweighting to Obtain Canonical Distributions and Ensemble Averages. J. Chem. Theory Comput. 2012, 8, 3777-3792.

(29) Gao, Y. Q. An integrate-over-temperature approach for enhanced sampling. J. Chem. Phys. 2008, 128, 064105.

(30) Yang, L.; Gao, Y. Q. A selective integrated tempering method. J. Chem. Phys. 2009, $131,214109$.

(31) Yang, Y. I.; Zhang, J.; Che, X.; Yang, L.; Gao, Y. Q. Efficient sampling over rough energy landscapes with high barriers: A combination of metadynamics with integrated tempering sampling. J. Chem. Phys. 2016, 144, 094105.

(32) Yang, M.; Yang, L.; Gao, Y.; Hu, H. Combine umbrella sampling with integrated tempering method for efficient and accurate calculation of free energy changes of complex energy surface. J. Chem. Phys. 2014, 141, 044108.

(33) Xie, L.; Shen, L.; Chen, Z.-N.; Yang, M. Efficient free energy calculations by combining two complementary tempering sampling methods. J. Chem. Phys. 2017, 146, 024103. 
(34) Sun, G.; Jiang, H. Ab initio molecular dynamics with enhanced sampling for surface reaction kinetics at finite temperatures: $\mathrm{CH}_{2} \leftrightarrow \mathrm{CH}+\mathrm{H}$ on $\mathrm{Ni}(111)$ as a case study. J. Chem. Phys. 2015, 143, 234706.

(35) Chen, Z.-N.; Shen, L.; Yang, M.; Fu, G.; Hu, H. Enhanced Ab Initio Molecular Dynamics Simulation of the Temperature-Dependent Thermodynamics for the Diffusion of Carbon Monoxide on $\mathrm{Ru}(0001)$ Surface. J. Phys. Chem. C 2015, 119, 26422-26428.

(36) Lionel, R.; Ranga, K.; Martin, H. Neural networks in chemical reaction dynamics; Oxford University Press: New York, 2012.

(37) Behler, J.; Parrinello, M. Generalized neural-network representation of highdimensional potential-energy surfaces. Phys. Rev. Lett. 2007, 98, 146401.

(38) Behler, J. Constructing high-dimensional neural network potentials: A tutorial review. Int. J. Quantum Chem. 2015, 115, 1032-1050.

(39) Manzhos, S.; Dawes, R.; Carrington, T. Neural network-based approaches for building high dimensional and quantum dynamics-friendly potential energy surfaces. Int. J. Quantum Chem. 2015, 115, 1012-1020.

(40) Fu, B.; Chen, J.; Liu, T.; Shao, K.; Zhang, D. H. Highly Accurately Fitted Potential Energy Surfaces for Polyatomic Reactive Systems. Acta Phys.-Chim. Sin. 2019, 35, $145-157$.

(41) Ma, S.; Liu, Z.-P. Machine Learning for Atomic Simulation and Activity Prediction in Heterogeneous Catalysis: Current Status and Future. ACS Catal. 2020, 10, 1321313226.

(42) Shetty, S.; Jansen, A. P. J.; van Santen, R. A. Direct versus Hydrogen-Assisted CO Dissociation. J. Am. Chem. Soc. 2009, 131, 12874-12875. 
(43) Li, H. P.; Fu, G.; Xu, X. A new insight into the initial step in the Fischer-Tropsch synthesis: CO dissociation on Ru surfaces. Phys. Chem. Chem. Phys. 2012, 14, 1668616694.

(44) Cheng, K.; Kang, J. C.; King, D. L.; Subramanian, V.; Zhou, C.; Zhang, Q. H.; Wang, Y. In Advances in Catalysis; Song, C., Ed.; Elsevier Academic Press Inc: San Diego, 2017; Vol. 60; pp 125-208.

(45) Hansen, H. A.; Varley, J. B.; Peterson, A. A.; Norskov, J. K. Understanding Trends in the Electrocatalytic Activity of Metals and Enzymes for $\mathrm{CO}_{2}$ Reduction to CO. J. Phys. Chem. Lett. 2013, 4, 388-392.

(46) Chen, S.; Chen, Z.-N.; Fang, W.-H.; Zhuang, W.; Zhang, L.; Zhang, J. Ag Ag $_{10}{ }^{-}$ Oxo Cluster Containing Single-Atom Silver Sites: Atomic Structure and Synergistic Electronic Properties. Angew. Chem. Int. Ed. 2019, 58, 10932-10935.

(47) Jin, S.; Hao, Z.; Zhang, K.; Yan, Z.; Chen, J. Advances and Challenges for the Electrochemical Reduction of $\mathrm{CO}_{2}$ to $\mathrm{CO}$ : From Fundamentals to Industrialization. Angew. Chem. Int. Ed. 2021, doi:10.1002/anie.202101818.

(48) Xu, Z.-N.; Sun, J.; Lin, C.-S.; Jiang, X.-M.; Chen, Q.-S.; Peng, S.-Y.; Wang, M.-S.; Guo, G.-C. High-Performance and Long-Lived Pd Nanocatalyst Directed by Shape Effect for CO Oxidative Coupling to Dimethyl Oxalate. ACS Catal. 2013, 3, 118-122.

(49) Tan, H.-Z.; Chen, Z.-N.; Xu, Z.-N.; Sun, J.; Wang, Z.-Q.; Si, R.; Zhuang, W.; Guo, G.-C. Synthesis of High-Performance and High-Stability $\mathrm{Pd}(\mathrm{II}) / \mathrm{NaY}$ Catalyst for CO Direct Selective Conversion to Dimethyl Carbonate by Rational Design. ACS Catal. 2019, 9, 3595-3603.

(50) Wang, Z. Q.; Sun, J.; Xu, Z. N.; Guo, G. C. CO direct esterification to dimethyl oxalate and dimethyl carbonate: the key functional motifs for catalytic selectivity. Nanoscale 2020, 12, 20131-20140. 
(51) Hammer, B.; Norskov, J. K. Theoretical surface science and catalysis - Calculations and concepts. Adv. Catal. 2000, 45, 71-129.

(52) Perdew, J. P.; Burke, K.; Ernzerhof, M. Generalized gradient approximation made simple. Phys. Rev. Lett. 1996, 77, 3865.

(53) Kresse, G.; Furthmüller, J. Efficient iterative schemes for ab initio total-energy calculations using a plane-wave basis set. Phys. Rev. B 1996, 54, 11169-11186.

(54) Kresse, G.; Furthmüller, J. Efficiency of ab-initio total energy calculations for metals and semiconductors using a plane-wave basis set. Comput. Mater. Sci. 1996, 6, 15-50.

(55) Blöchl, P. E. Projector augmented-wave method. Phys. Rev. B 1994, 50, 17953-17979.

(56) Monkhorst, H. J.; Pack, J. D. Special points for Brillouin-zone integrations. Phys. Rev. B 1976, 13, 5188-5192.

(57) Chen, J.; Xu, X.; Xu, X.; Zhang, D. H. A global potential energy surface for the $\mathrm{H}_{2}+$ $\mathrm{OH} \leftrightarrow \mathrm{H}_{2} \mathrm{O}+\mathrm{H}$ reaction using neural networks. J. Chem. Phys. 2013, 138, 154301.

(58) Martyna, G. J.; Klein, M. L.; Tuckerman, M. Nosé-Hoover chains: The canonical ensemble via continuous dynamics. J. Chem. Phys. 1992, 97, 2635-2643.

(59) Martyna, G. J.; Tuckerman, M. E.; Tobias, D. J.; Klein, M. L. Explicit reversible integrators for extended systems dynamics. Mol. Phys. 1996, 87, 1117-1157.

(60) Swope, W. C.; Andersen, H. C.; Berens, P. H.; Wilson, K. R. A computer simulation method for the calculation of equilibrium constants for the formation of physical clusters of molecules: Application to small water clusters. J. Chem. Phys. 1982, 76, 637-649.

(61) Tuckerman, M.; Berne, B. J.; Martyna, G. J. Reversible multiple time scale molecular dynamics. J. Chem. Phys. 1992, 97, 1990-2001. 
(62) Over, H.; Moritz, W.; Ertl, G. Anisotropic atomic motions in structural analysis by low energy electron diffraction. Phys. Rev. Lett. 1993, 70, 315-318.

(63) Noei, H.; Kozachuk, O.; Amirjalayer, S.; Bureekaew, S.; Kauer, M.; Schmid, R.; Marler, B.; Muhler, M.; Fischer, R. A.; Wang, Y. CO Adsorption on a Mixed-Valence Ruthenium Metal-Organic Framework Studied by UHV-FTIR Spectroscopy and DFT Calculations. J. Phys. Chem. C 2013, 117, 5658-5666.

(64) Pfnür, H.; Menzel, D.; Hoffmann, F.; Ortega, A.; Bradshaw, A. High resolution vibrational spectroscopy of $\mathrm{CO}$ on $\mathrm{Ru}(001)$ : The importance of lateral interactions. Surf. Sci. 1980, 93, 431-452.

(65) Hoffmann, F. M.; Persson, B. N. J. Vibrational dephasing of terminally bonded CO on Ru(001). Phys. Rev. B 1986, 34, 4354-4357.

(66) Jakob, P.; Persson, B. N. J. Dephasing of localized and delocalized vibrational modes: CO adsorbed on Ru(001). Phys. Rev. B 1997, 56, 10644-10650.

(67) Vattulainen, I.; Merikoski, J.; Ala-Nissila, T.; Ying, S. C. Non-Arrhenius Behavior of Surface Diffusion near a Phase Transition Boundary. Phys. Rev. Lett. 1997, 79, 257260.

(68) Pentcheva, R.; Fichthorn, K. A.; Scheffler, M.; Bernhard, T.; Pfandzelter, R.; Winter, H. Non-Arrhenius Behavior of the Island Density in Metal Heteroepitaxy: Co on $\mathrm{Cu}(001)$. Phys. Rev. Lett. 2003, 90, 076101.

(69) Zhang, I. Y.; Xu, X. Reaching a Uniform Accuracy for Complex Molecular Systems: Long-Range-Corrected XYG3 Doubly Hybrid Density Functional. J. Phys. Chem. Lett. 2013, 4, 1669-1675.

(70) Zhang, I. Y.; Xu, X. Simultaneous Attenuation of Both Self-Interaction Error and 
Nondynamic Correlation Error in Density Functional Theory: A Spin-Pair Distinctive Adiabatic-Connection Approximation. J. Phys. Chem. Lett. 2019, 10, 2617-2623.

(71) Chen, Z.-N.; Shen, T.; Wang, Y.; Zhang, I. Y. Accurate Description of Catalytic Selectivity: Challenges and Opportunities for the Development of Density Functional Approximations. CCS Chem. 2021, 3, 136-143.

(72) Zhang, I. Y.; Xu, X. Exploring the Limits of the XYG3-Type Doubly Hybrid Approximations for the Main-Group Chemistry: The xDH@B3LYP Model. J. Phys. Chem. Lett. 2021, 12, 2638-2644. 